\title{
Telerehabilitation services: A successful paradigm for occupational therapy clinical services?
}

\begin{abstract}
This review sheds light on telehealth and telerehabilitation services with a focus in occupational therapy practice. It summarizes clinical and research applications, benefits and impact, limitations, barriers and current needs, and recommendations for future adoption of more evidence-based occupational therapy telehealth services. Our review confirmed that telehealth is useful and can be an appropriate remote service delivery model for occupational therapy. Telehealth connects clients with occupational therapists through information and communication technologies, thereby improving easy access to services for underserved populations when in-person encounters are not possible or practical. Telerehabilitation provides occupational therapists and other healthcare professionals with flexibility to continue treating their patients while following best practices and ethical standards. Telehealth is needed nowadays more than ever and more research is recommended to further address current and future challenges and support expansion of telehealth applications in the field of rehabilitation and occupational therapy.
\end{abstract}

Keywords: telehealth, telerehabilitation, teletherapy, telemedicine, rehabilitation, service delivery, occupational therapy
Volume 5 Issue 2 - 2020

Hassan Izzeddin Sarsak

Occupational Therapy Program, Batterjee Medical College, Saudi Arabia

Correspondence: Hassan Izzeddin Sarsak (PhD, OT), Occupational Therapy Program, Batterjee Medical College, Jeddah, Saudi Arabia, Email hassan.sarsak@bmc.edu.sa

Received: April 04, 2020 | Published: April I6, 2020

\section{Introduction}

Telehealth is the use of electronic information and telecommunications technologies to support long-distance clinical health care, patient and professional health-related education, public health and health administration. ${ }^{1}$ It is the provision of health care remotely by means of a variety of telecommunication tools, including telephones, smartphones, and mobile wireless devices, with or without a video connection. Telehealth is growing rapidly and has the potential to transform the delivery of health care for millions of persons. Telerehabilitation (TR) is a relatively new and developing field of telehealth. TR is the use of telecommunication technology to deliver and support rehabilitation services ${ }^{2}$ and it is the clinical application of consultative, preventative, diagnostic, and therapeutic services via two-way or multi-point interactive telecommunication technology. TR refers to the delivery of rehabilitation services via information and communication technologies. These services include but not limited to assessment, monitoring, intervention, supervision, education, consultation, and counseling. ${ }^{3}$ There are two main components of TR services: rehabilitation service (clinical application) and telecommunication/information technology. This is a way to interact, examine, diagnose, and treat patients remotely.

Research reported many specific advantages and disadvantages for TR. Advantages of TR include practitioners and patients no longer have to be in the same room, the barrier of distance can be minimized to deliver rehabilitation interventions remotely across the continuum of care, decreased travel to specialized urban health centers, enhanced clinical support in local communities, improved access to specialized services, educational benefits, reduced feelings of isolation for rural clinicians, and improved service stability and multimedia communication. Disadvantages of TR include difficulties encountered by the user as well as with the equipment. User problems may be classified as cognitive, motor, and social. In terms of cognitive factors, technological and communication devices may be extremely difficult for an individual with a neurological disability to operate. For example, many brain injuries involve disturbances in an individual's ability to plan, organize, initiate activities, control impulses, concentrate, problem solve, and recall information. ${ }^{4}$

Although several reviews have examined the historical use and effects of telehealth, ${ }^{5,6}$ few articles have characterized its current status. Here we review the current trends of telehealth and telerehabilitation, clinical and research applications, their benefits and impact, their limitations, barriers and needs, and recommendations for the current and future adoption of telehealth services with a concentration in evidence-based occupational therapy practice.

\section{Telehealth and telerehabilitation: clinical and research applications}

Common approach to building an information technology (IT) infrastructure to support TR is to purchase IT systems that create similar experiences with traditional face-to-face encounters. Example are but not limited to: videoconferencing system, virtual reality, and electronic/portable devices. Essential TR Technology components include human factors (patients, providers, organizations, and society), economic factors, and technologies. Figures 1 demonstrates a visual representation of TR model with all integrated essential elements.

TR, compared to traditional rehabilitation services, helps decrease travel to specialized health centers and improve easy access to a variety of clinical services (Figure 2).

\section{Practical considerations: how to choose the right tool?}

How do you select the technology to provide telerehabilitation services? In order to answer this question, we need to: identify needed services and desirable features, balance real world trade offs, and choose the simplest and least expensive technology that meets the client's needs. Figures 3 and 4 show examples of devices used in TR (Figure 3) and sample clinical implications for remote clinical services utilized to enhance cognitive and physical abilities (Figure 4). 


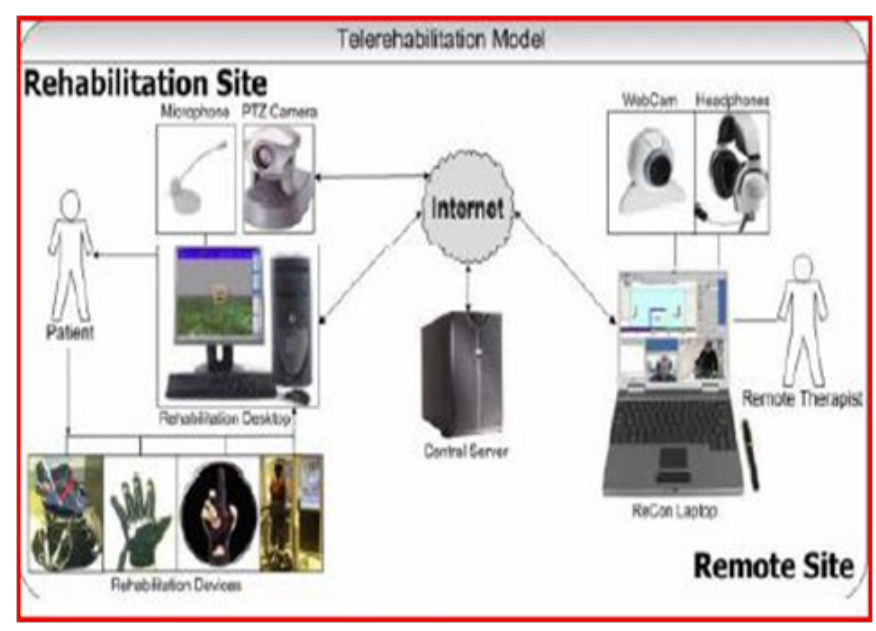

Figure ITelerehabilitation (TR) model.

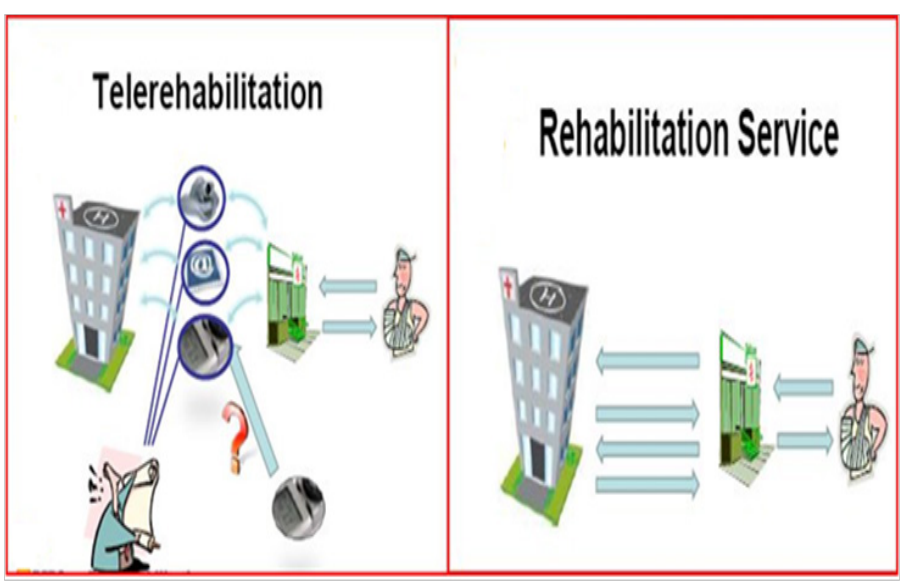

Figure 2 Telerehabilitation (TR) versus traditional rehabilitation services.

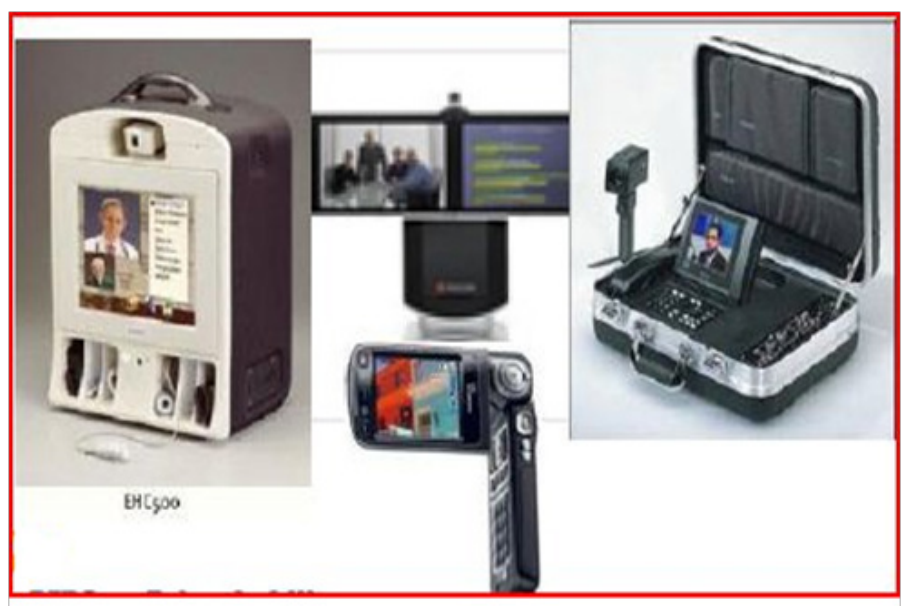

Figure 3 Examples of devices used in TR.

\section{Methods}

\section{Review of literature, search strategy, and method used}

A research has been made in the following databases: Ovid,
PsychINFO, MEDLINE, Global Health, CINAHL, and PubMed. Keywords and Search items used to search articles for our study were telehealth, telerehabilitation, teletherapy, telemedicine, rehabilitation, service delivery, and occupational therapy. For the purpose of this study, we created a clinical/research PICO question (Population, Intervention, Comparison, and Outcome), a key to evidencebased decision. ${ }^{8} \mathrm{~A}$ thorough review was conducted to identify most relevant and recent evidence-based research related to telehealth and telerehabilitation services with a focus in occupational therapy practice. Throughout the article, evidence-based occupational therapy telerehabilitation services and interventions are emphasized. Articles were selected based on our PICO question and based on the hierarchy of levels of evidence in evidence based practice of well-designed research studies. ${ }^{9}$ The PICO formed for our study is as follows:

(P): For persons with different diagnoses and from different age groups

(I): Are telerehabilitation occupational therapy clinical services

(C): Compared to traditional occupational therapy services

(O): Effective and successful paradigm as an alternative service delivery model?

\section{Results}

\section{Telerehabilitation: evidence-based occupational therapy services}

Trelerehabilitation have been applied in a variety of clinical and research settings with different diagnoses and age groups and proved to be reliable and effective in that venue. A study of 98 adults with mobility impairments using wheeled mobility and seating devices (manual wheelchair, power wheelchair, scooter) were tested to determine whether or not the telerehabilitation (TR) treatment condition at remote clinics was equally effective when compared to the standard in-person (IP) treatment at local clinics. They found that the telerehabilitation treatment condition was equally effective for most functional items tested. Another study of 46 subjects with mobility impairments using wheeled mobility and seating devices evaluated the interrater reliability between a generalist clinician in person (IP) and an expert clinician observing through Telerehabilitation (TR) from a remote clinic. The expert clinician, located more than 100 miles away, was able to accurately evaluate the functional mobility needs of clients being assessed for new mobility devices. ${ }^{10}$

In addition, a multisite randomized controlled clinical trial was completed with 99 people $<6$ months after stroke who had limited access to formal therapy. Participants were randomized into one of two groups, (1) a home exercise program or (2) a robot-assisted therapy + home exercise program, and participated in an 8-wk home intervention. Results of this study concluded that a robot-assisted intervention coupled with a home exercise program and a home exercise program alone administered using a telerehabilitation model may be valuable approaches to improving quality of life and depression in people after stroke. ${ }^{11}$ Hermann et al. ${ }^{12}$ examined the efficacy of a remotely based arm rehabilitation regimen. A 62-year-old man participated in occupation-based, task-specific practice of activities of daily living $($ ADLs) $>3$ years after stroke. The entire regimen was administered over the Internet using personal computer-based cameras and free network meeting software. The participant exhibited reduced impairment and reduced functional limitation. He also expressed 
enhanced satisfaction with his ability to perform ADLs and rated his ADL performance better after intervention. The participant could drive using both hands, use eating utensils, and catch and throw a ball. Data from this study suggested feasibility and efficacy of a remotely based, inexpensive approach using functional electrical stimulation for affected arm rehabilitation after stroke. ${ }^{12}$ Also, a previous study provided a detailed design description of a telerehabilitation system for arm and hand therapy following stroke. In this study, the system consisted of a Web-based library of status tests, therapy games, and progress charts, and could be used with a variety of input devices, including a low-cost force-feedback joystick capable of assisting or resisting in movement. Data from home-based usage by a chronic stroke subjects demonstrated the feasibility of using the system to direct a therapy program. The system mechanically assisted in movement, and was able to track improvements in movement ability. ${ }^{13}$
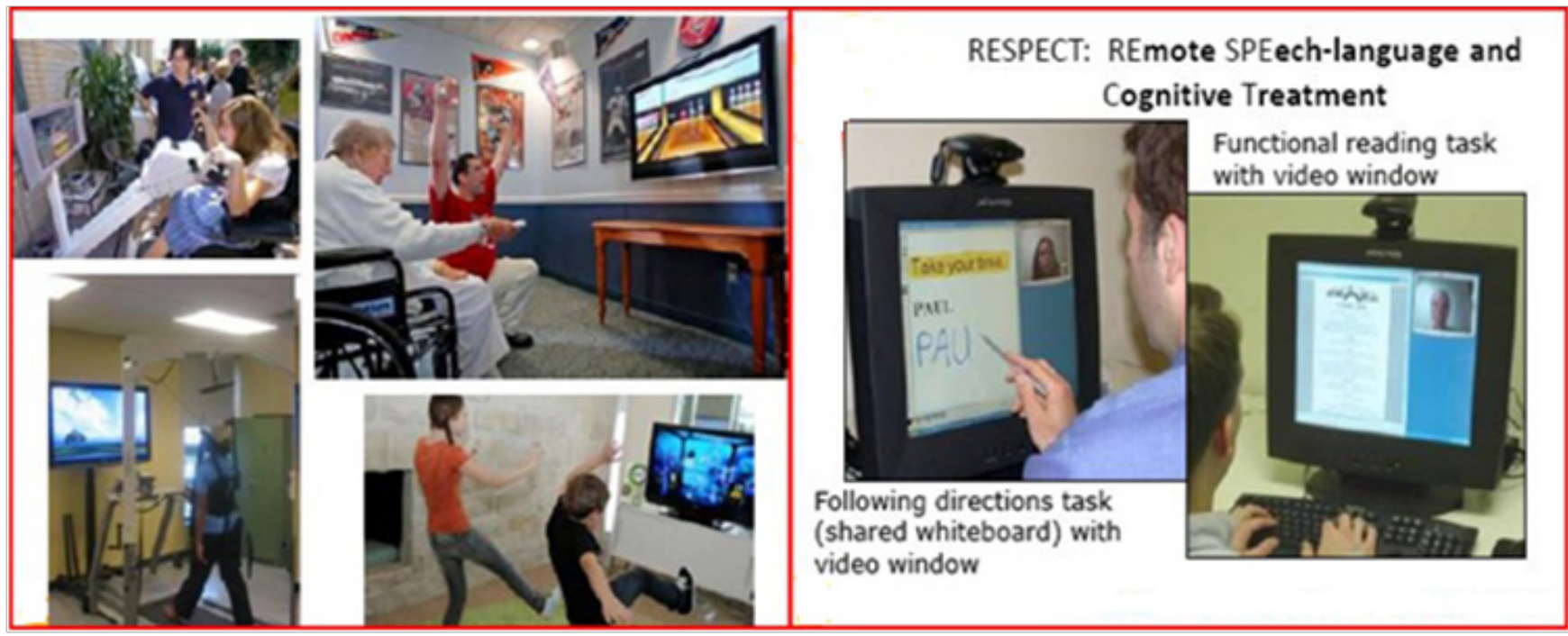

Figure 4 Sample TR clinical implications.

Furthermore, a pilot series of three case studies with 3-month follow-up was conducted to explore the feasibility of implementing the Cognitive Orientation to daily Occupational Performance approach (CO-OP) in a telerehabilitation format and to examine its impact on community integration and executive dysfunction for adults with traumatic brain injury (TBI). Three adults (all males, $>10$ years post-TBI) and their significant others were recruited. The COOP intervention, a meta-cognitive approach, was delivered through videoconferencing via Internet to train three of five participantidentified goals. This study concluded that telerehabilitation shows promise as a way to deliver the $\mathrm{CO}-\mathrm{OP}$ approach and may help promote community integration of individuals living with TBI. ${ }^{14}$

Additionally, telerehabilitation applications in occupational therapy were also explored in Children and adolescents. A school-based rehabilitation program included the implementation of occupational therapy services via two-way interactive videoconferencing technology. The participants included in this program were children, ages 6 to 11 years, who attended an online charter school and had difficulties in the areas of fine motor and/or visual motor skills which impacted success with handwriting. Each participant completed a virtual evaluation and six 30-minute intervention sessions. Outcomes revealed improvements in handwriting performance for most students who participated in the program and high satisfaction rates reported by all participants. ${ }^{15} \mathrm{~A}$ previous research project explored the use of telerehabilitation for collaborative occupational therapy sessions with parents of children with autism spectrum disorders (ASD). The aim was to improve carryover of therapeutic strategies by parents to address children's sensory modulation in their natural environments. Four families participated in clinic-based sessions with the therapist followed by online sessions for six weeks. Data consisted of family schedules, sensory diets, archived webcam sessions, and sensory processing measure home form scores before and after initiation of the telerehabilitation sessions. Results demonstrated the potential for using telerehabilitation as a tool to provide collaborative occupational therapy in order to improve carryover of home programs for children with ASD by providing opportunities for parents to ask questions, review sensory techniques, and understand the therapist's clinical reasoning. ${ }^{16} \mathrm{Cason}^{17}$ explored the use of telerehabilitation as an alternative service delivery model for early intervention therapy services applying the enTECH Telerehabilitation Program. Utilizing the Kentucky Telehealth Network, two families living in rural Kentucky received occupational therapy services over a 12-week period. Following program implementation, qualitative data was collected using participant journals and interviews. Results of the program evaluation indicated that telerehabilitation has the potential to cost-effectively meet the therapeutic needs of children living in rural areas where provider shortages exist. The enTECH Telerehabilitation Program serves as a model for how telerehabilitation can be used to deliver early intervention services and improve access to rehabilitation services. ${ }^{17}$

For adolescents, A 3-month proof-of-concept pilot study investigated whether in-home remotely monitored virtual reality videogame-based telerehabilitation in adolescents with hemiplegic cerebral palsy can improve hand function and forearm bone health, and demonstrate alterations in motor circuitry activation. Virtual reality videogame-based rehabilitation systems were installed in the homes of 3 adolescents with severe hemiplegic cerebral palsy and networked via secure Internet connections to the collaborating 
engineering school and children's hospital. Participants were asked to exercise the plegic hand 30 minutes a day, 5 days a week using a sensor glove fitted to the plegic hand and attached to a remotely monitored videogame console installed in their home. Games were custom developed, focused on finger movement, and included a screen avatar of the hand. All 3 adolescents showed improved function of the plegic hand on occupational therapy testing, including increased ability to lift objects, and improved finger range of motion (ROM) based on remote measurements. Use of remotely monitored virtual reality videogame telerehabilitation appeared to produce improved hand function and forearm bone health in adolescents with chronic disability who practice regularly. ${ }^{18}$

Telerehabilitation technologies have been integrated into occupational therapy services and proved to be useful with older adults. A study investigated the feasibility of providing telerehabilitation in the home as an alternative to conventional ambulatory rehabilitation. Telerehabilitation employed a coaching model, with fewer therapist home visits, more feedback and homework for the patient. Patients had a tablet computer loaded with a videoconferencing application to connect with therapists and relevant therapeutic applications for 8 weeks. On average participants felt that they had achieved $75 \%$ of the goals set at the beginning of the program and high levels of satisfaction were recorded. They concluded that telerehabilitation using off-theshelf technology is feasible for post-acute treatment. ${ }^{19}$ Previous studies evaluated a telerehabilitation program called the Low Activities of Daily Living Monitoring Program (LAMP). Patients in this program received adaptive equipment for use in the home. LAMP healthcare providers monitored their patients remotely and provided home environmentl interventions such as self-management strategies and environmental modifications. The LAMP was able to deliver rehabilitation services for patients in their homes efficiently. It was cost-effective and decreased in hospital and nursing stays. ${ }^{20,21}$

\section{Discussion}

This review article furnishes an overview of the evidence for telehealth use in occupational therapy, discusses key considerations and practical implications, and summarizes current and potential future trends for practitioners in the use of telehealth.

Over the past two decades, the practice of occupational therapy has been increasingly influenced by technological advances in the use of information and communication technologies (ICT) and associated changes in health care policy. Emergent from this evolution is the application of telehealth and telerehabilitation to deliver occupational therapy services to a client who is in a different physical location than the provider. ${ }^{22} \mathrm{~A}$ variety of terms have been used to describe this remote service delivery model, such as: tele-occupational therapy, telerehabilitation, teletherapy, telecare, telemedicine, and telepractice. The World Federation of Occupational Therapists (WFOT) ${ }^{23}$ and the World Report on Disabilities co-produced by the World Health Organization (WHO) affirmed the efficacy of telehealth and telerehabilitation for the delivery of rehabilitation and occupational therapy services stating its use "leads to similar or better clinical outcomes when compared to conventional interventions". ${ }^{24}$

Telerehabiltation has a positive significant impact on patient's overall health and helps develop functional performance. This impact was summarized in what is called (the 10 "E's"). ${ }^{25}$ The 10 "E's" of any "Tele" activity are represented in Table 1 .
Table I The 10 "E's" of any "telehealth" service.

\begin{tabular}{l} 
Any successful telehealth activity should adhere to: \\
\hline I.Efficiency (decrease costs) \\
2.Enhancing quality of care (client to provider, access to outcomes and \\
specialists) \\
3.Evidence-based practice \\
4.Empowering consumers \\
5.Encouraging new relationships \\
6.Education \\
7.Enabling information exchange \\
8.Extension \\
9.Ethics and Equity \\
10.Easy to use, Entertaining, and Exciting opportunities
\end{tabular}

\section{Ethical considerations}

Occupational therapists (OTs) using telerehabilitation methods must adhere to the WFOT occupational therapy code of ethics and maintain and comply with the standards of practice, ensuring both their proficiencies as practitioners and the well-being of their clients. ${ }^{26}$ The WFOT provided the occupational therapy telehealth ethical considerations ${ }^{23}$ which are summarized in (Table 2).

\section{COVID-I9 has put telehealth on the front lines!}

Nowadays, with the digital revolution, telehealth is evolving from clinics to the home. The newly discovered Coronavirus (COVID-19) and social distancing has put telehealth on the front lines. As telehealth becomes imperative in the midst of the COVID19 pandemic, occupational therapy professionals have been working and advocating to make this remote health option even more available in most of the rehabilitative fields and services. Telemedicine has been put into focus since social distancing guidelines have been put in place, offering patients the opportunity to get the care they need via telecommunications. Many clinical settings have been trying their best to help expand telemedicine services for patients during these times. While medical care centers are considered essential under current quarantine regulations, remote visits, otherwise known as telehealth, are playing an important role in keeping patients out of clinics unless absolutely necessary. Helping with telehealth efforts by rapidly expanding the availability of video visits for both scheduled and urgent-care visits have been put into action. Video visits help keep patients and providers safe while allowing hospitals to have more critical protective equipment when COVID-19 infected people need care at a hospital.

Despite the expansion of telehealth in recent years, health experts say more medical fields need to be included, including occupational therapy, physical therapy, speech-language pathology and audiology. This should be the focus as social distancing becomes the norm to curb the rise of COVID-19 cases. Telehealth helps maintain progress made in patients' health until patients can meet with providers again in person. Unfortunately, we really see the focus on physician-type 
services, but not so much on therapeutic-based practices. Occupational therapists are classified as essential health care providers and applying telehealth focus on not losing any progress patients have made. However, many governmental and private insurance companies don't reimburse therapeutic-based telemedicine. Therefore, support and legislations that would allow for some flexibility in reimbursement by insurers to patients who rely on therapeutic telemedicine such as occupational therapy services are crucial. It is now the time to demonstrate the value of telehealth for doctors and patients. We are really able to maintain the health and wellness of society as a whole, and that's valuable in thinking about what the change in health care delivery is going to look like as we move forward, even past COVID-19. Practitioners and patients can see for themselves if telehealth is effective while practicing social distancing practices.

Table 2 WFOT occupational therapy telehealth ethical considerations.

\begin{tabular}{ll} 
Ethical consideration & Explanation \\
\hline Licensure/Registration & - Occupational therapists (OTs) shall comply with professional licensure/registration requirements. \\
& $\begin{array}{l}\text { Collaboration with Local } \\
\text { Occupational Therapists }\end{array} \quad \begin{array}{l}\text { therapy providers, organizations, educationalinstitutions, and/or associations in the interest of cohesive, relevant, } \\
\text { andsustainable services. }\end{array}$
\end{tabular}

Client Selection

- OTs should use clinical reasoning to determine the appropriateness of telehealth use based on individual client situations (e.g., client's diagnosis andimpairments, nature of the occupational therapy interventions to be provided, client's ability to access technologies, etc.).

- Telehealth should therefore not be used to avoid in-person services when indicated by client-specific needs, nor be usedby therapists to avoid contact with clients on the basis of discrimination.

Consent to Treat

Professional Liability Insurance

Confidentiality

Personal and Cultural Attributes

Provider Competence/ Standard of Care

Reimbursement/Payer Guidelines

Authentic Occupational Therapy Practice
- OTs shall inform clients about the nature of the occupational therapy services to be provided, risks, benefits, alternate treatment options, and any limits to protection of privacy, security, and confidentiality of personal health information associated with the technology.

- OTsshould comply with jurisdictional, institutional, and professional requirements for maintaining professional liability insurance.

- OTs should confirm coverage of professionalliability insurance for the geographic areas served.

- Users of telehealth are obligated to employ mechanisms to ensureconfidentiality for synchronous and stored client data in compliance withjurisdictional, institutional, and professional regulations and policiesgoverning occupational therapy practice.

- Therapists should follow principles outlined in the WFOT's document, Guiding Principles on Diversity and Culture and the Diversity and Culture position statement.

- Therapists must maintain professional competency, acquire competency using telehealth technologies, ensure client safety, and adhere to ethical principles of practice.

- Therapists must adhere to reimbursement requirements and accurately represent the services delivered through telehealth.

- The WFOT endorses practice that is client-centered and occupation-centered, and which portrays the breadth of the profession.

\section{Barriers/limitations and solutions/future recommendations}

Though the use of telehealth and telerehabilitation is promising and expanding quickly as a useful and helpful method of service delivery for many healthcare providers, there remain issues of efficacy, cost, reimbursement, practitioner competence, and regulations. In addition, recent studies showed that ease of use, usability, and accessibility for telerehabilitation services could be sometimes unsolved problems, especially for older people and people who live in rural areas who may have little experience or confidence in using advanced technology ${ }^{27}$ There is a significant need for occupational therapists to document, research, and publish on the efficacy and effectiveness of clinical services provided using telehealth and telerehabilitation technologies. Telerehabilitation research needs larger and more comprehensive analyses of patient outcomes and a variety of clinical outcome measures used in telerehabilitation are needed. Further future studies such as cost-effectiveness, longitudinal observational studies, and randomized clinical trials are recommended, could be focus areas, and may have the potential for more helpful and useful telehealth-related outcomes. Evidence-based practice would help raise awareness, develop clinician \& client education, and support rationale 
of using telerehabilitation services for justification and reimbursement purposes.

\section{Conclusion}

Telehealth is useful and can be an appropriate remote service delivery model for occupational therapy. Telehealth technologies may improve access to occupational therapy services for underserved populations such as older adults and those who live in rural areas when in-person encounters are not possible or practical. Telerehabilitation provides occupational therapists and other healthcare providers flexibility to continue treating their patients while following best practices and ethical standards. Telehealth is needed nowadays more than ever and more research is recommended to further support and advocate for telehealth applications.

\section{Acknowledgments}

None.

\section{Conflicts of interest}

The authors declare have no conflict of interest about the publication of this paper.

\section{References}

1. Dorsey ER, Topol EJ. State of telehealth. NEngl J Med. 2016;375:15461.

2. Theodoros D, Russell T, Latifi R. Telerehabilitation: current perspectives. Studies in health technology and informatics. 2008;131: 191-210.

3. Fairman A, Brickner A, Lieberman D, et al. Telerehabilitation. The American Journal of Occupational Therapy. 2010;64(6):S92.

4. Torsney K. Advantages and disadvantages of telerehabilitation for persons with neurological disabilities. NeuroRehabilitation. 2003;18(2):183-185.

5. Ekeland AG, Bowes A, Flottorp S. Effectiveness of telemedicine: A systematic review of reviews. Int J Med Inform. 2010;79:736-771.

6. McLean S, Sheikh A, Cresswell K, et al. The impact of telehealthcare on the quality and safety of care: A systematic overview. PloS one. $2013 ; 8(8)$.

7. Wootton R. Twenty years of telemedicine in chronic disease management: An evidence synthesis. J Telemed Telecare. 2012;18(4): 211-220.

8. Richardson WS, Wilson MC, Nishikawa J, et al. The well-built clinical question: a key to evidence-based decisions. Acp $j$ club. 1995;123(3):A12-13.

9. Hughes I. Action research in healthcare: What is the evidence?. ALAR Journal. 2006;11(1):29-39.

10. Sarsak HI. Functional assessment of wheeled mobility and seating interventions: Relationship of self-report and performance-based assessments. American Journal of Biomedical Science and Research. Arcadia, CA: BiomedGrid. 2019.

11. Linder SM, Rosenfeldt AB, Bay RC, et al. Improving quality of life and depression after stroke through telerehabilitation. American Journal of Occupational Therapy. 2015;69(2).
12. Hermann VH, Herzog M, Jordan R, et al. Telerehabilitation and electrical stilation: An occupation-based, client-centered stroke intervention. American Journal of Occupational Therapy. 2010;64(1):73-81.

13. Reinkensmeyer DJ, Pang CT, Nessler JA, et al. Web-based telerehabilitation for the upper extremity after stroke. IEEE transactions on neural systems and rehabilitation engineering. 2002;10(2):102-108.

14. Ng EM, Polatajko HJ, Marziali E, et al. Telerehabilitation for addressing executive dysfunction after traumatic brain injury. Brain Injury. 2013;27(5):548-564.

15. Criss MJ. School-based telerehabilitation in occupational therapy: Using telerehabilitation technologies to promote improvements in student performance. International journal of telerehabilitation. 2013;5(1):39.

16. Gibbs V, Toth-Cohen S. Family-centered occupational therapy and telerehabilitation for children with autism spectrum disorders. Occupational Therapy in Health Care. 2011; 25(4):298-314.

17. Cason J. A pilot telerehabilitation program: Delivering early intervention services to rural families. International Journal of Telerehabilitation. 2009;1(1):29.

18. Golomb MR, McDonald BC, Warden SJ, et al. In-home virtual reality videogame telerehabilitation in adolescents with hemiplegic cerebral palsy. Archives of physical medicine and rehabilitation. 2010;91(1):18.

19. Crotty M, Killington M, van den Berg M, et al. Telerehabilitation for older people using off-the-shelf applications: acceptability and feasibility. Journal of telemedicine and telecare. 2014;20(7):370-376.

20. Bendixen RM, Levy CE, Olive ES, et al. Cost effectiveness of a telerehabilitation program to support chronically ill and disabled elders in their homes. Telemedicine and e-Health. 2009;15(1):31-38.

21. Bendixen RM, Horn K, Levy C. Using telerehabilitation to support elders with chronic illness in their homes. Topics in Geriatric Rehabilitation. 2007;23(1):47-51.

22. Cason J. Telehealth: A rapidly developing service delivery model for occupational therapy. International Journal of Telerehabilitation. 2004;6(1):29.

23. World federation of occupational therapists [WFOT]. (2005). Code of ethics.

24. Occupational Therapists WFO. World federation of occupational therapists' position statement on telehealth. International journal of telerehabilitation. 2014.

25. Cherry JC, Moffatt TP, Rodriguez C, et al. Diabetes disease management program for an indigent population empowered by telemedicine technology. Diabetes Technol Ther. 2002;4(6):783-791.

26. Wakeford L, Wittman PP, White MW, et al. Telerehabilitation position paper. The American Journal of Occupational Therapy. 2005;59(6):656-660.

27. Teixeira A, Pereira C, Silva MO, et al. New telerehabilitation services for the elderly. In Handbook of Research on ICTs and Management Systems for Improving Efficiency in Healthcare and Social Care. IGI Global. 109-132. 